\title{
A New Modification of the Chiron ACS Assay for Total Prostate-Specific Antigen Achieves Equimolar Response Characteristics and Improves the Detection of Prostate Cancer
}

\author{
Frank Oberpenning ${ }^{1 *}$, Christoph Weining ${ }^{1}$, Burkhard \\ Brandt $^{2}$, Gabriela De Angelis ${ }^{2}$, Achim Heinecke ${ }^{3}$, \\ Michael Hamm ${ }^{4}$, Petra Stieber ${ }^{5}$, Lothar Hertle ${ }^{1}$, \\ Hans-Peter Schmid ${ }^{6}$ and Axel Semjonow ${ }^{1}$ \\ 'Department of Urology, \\ 2Department of Clinical Chemistry and Laboratory Medicine, \\ 3Department of Medical Informatics and Biomathematics, \\ University of Münster, Münster, Germany \\ 4Department of Urology, Klinikum Augsburg, Germany \\ ${ }^{5}$ Department of Clinical Chemistry, Klinikum Grosshadern, \\ Ludwig-Maximilians-University, Munich, Germany \\ ${ }^{6}$ Department of Urology, Kantonsspital St. Gallen, St. Gallen, \\ Switzerland
}

Nonequimolar-response assays for prostate-specific antigen (PSA) are criticized for overestimating total PSA in some men without prostate cancer (PCA), and underestimating total PSA in some men with PCA. We recently studied three nonequimolar-response PSA assays that had undergone modifications. While two of the studied assays achieved equimolar-response characteristics with improved areas under receiver operating characteristic (ROC) curves (AUC), the modification of the Chiron ACS PSA assay (ACS PSA2, Chiron) failed to achieve this. Recently, the ACS assay underwent another modification (ACS PSA, Bayer), which we investigated.

Sera from 305 men (155 without and 150 with PCA, PSA $\geq \mathbf{2}$ and $\leq \mathbf{3 0} \boldsymbol{\mu g} / \mathrm{l}$, Tandem-E) were measured using both modifications of the ACS assay and equimolar-response reference methods (Tandem- $R$ free and Tandem-E, Hybritech). Molar response relative to the reference method and clinical performance (comparison of AUCs) between the previous and new ACS assay modifications were studied.

The new modification of the ACS assay (ACS PSA, Bayer) achieved equimolar-response characteristics but reported lower values (average 10\%) than the Tandem-E assay. Compared to the previous modification (ACS PSA2, Chiron), a 3\% improvement in AUC ( $p=$ 0.01) was found. Using results of the redesigned equimolar-response assay (ACS PSA, Bayer), we calculated that 6 of 155 men without PCA in this sample set could be spared unnecessary biopsy compared with the previous nonequimolar-response assay (ACS PSA2, Chiron) without missing additional PCA (90\% sensitivity).

These data provide additional evidence for clinical

*E-mail of the corresponding author: oberpen@uni-muenster.de advantages of equimolar-response over nonequimolar-response PSA assay formats. Clin Chem Lab Med 2003; 41(1):90-94

Key words: Prostate-specific antigen (PSA); Tumor markers; Prostatic neoplasms; Benign prostatic hyperplasia; Comparative study; Immunoassay; Protein isoforms; Reagent kits: Human.

Abbreviations: $\mathrm{AUC}$, area under curve; $\mathrm{Cl}$, confidence interval; PCA, prostate cancer; PSA, prostate-specific antigen; ROC, receiver operating characteristic; $S D$, standard deviation.

\section{Introduction}

As initially described by Graves (1) a skewed-response or nonequimolar-response assay for prostate-specific antigen (PSA) reports quantities of free PSA as greater than the same amount of PSA complexed to $\alpha_{1}$-antichymotrypsin. Therefore, nonequimolar-response assays may yield different values for specimens containing the same amount of total PSA but markedly different proportions of free PSA $(2,3)$. The major form of PSA in serum is that complexed to $\alpha_{1}$-antichymotrypsin $(4,5)$. This complexed form of PSA is commonly present in a higher proportion in men with prostate cancer (PCA) than in men with benign prostate conditions, who tend to have higher ratios of free over total PSA (5). Nonequimolar-response PSA assays have been criticized for their tendency to overestimate total PSA in men with benign prostatic hyperplasia, and underestimate total PSA in men with PCA.

In this context we recently studied three nonequimolar-response PSA assays that were modified by the manufacturers (Abbott, Wiesbaden, Germany; BioMérieux, Nürtingen, Germany; Chiron, Fernwald, Germany) (6). We reported on their performance before and after modification using 338 sera from men with benign and malignant prostates. All three assays showed a marked nonequimolar response prior to modification, among them the ACS PSA2 assay (Chiron). The ACS PSA2 assay (Chiron) represented a previous modification of the original ACS PSA assay by Ciba-Corning, which was among the first commercial PSA assays reported to have a nonequimolar-response since it detected the free form of PSA with a much higher sensitivity than the PSA- $\alpha 1$-antichymotrypsin complex (7-9). After modification the assays by Abbott and bioMérieux were found to produce an equimolar response and an improvement in the area under the receiver operating characteristic (ROC) curve (AUC) was 
observed. The modification of the ACS assay (ACS PSA2, Chiron) did not demonstrate equimolar-response assay characteristics and the AUC remained unchanged as compared with the original test (6). However, the modification of the ACS assay (ACS PSA2, Chiron) reported lower total PSA concentrations on testing identical samples (10).

More recently, the ACS PSA2 assay (Chiron) underwent another new modification and was renamed ACS PSA (Bayer, Fernwald, Germany), now distributed by Bayer Diagnostics. With this modification, the polyclonal-monoclonal antibodies (ACS PSA2, Chiron) were replaced by two monoclonal antibodies (ACS PSA, Bayer), which were adopted from the Bayer Immuno I assay. Here we report the changes in molar response characteristics and the diagnostic performance achieved by this new modification.

\section{Materials and Methods}

Of the 338 archival sera on which the preceding report (6) was based, serum aliquots from 305 men $(62$ men without clinical evidence of PCA, 93 patients with histologically benign prostate and 150 patients with untreated PCA) with a total PSA concentration of $\geq 2$ and $\leq 30 \mu \mathrm{g} / \mathrm{l}$ (mean 9.1; median 7.0; SD 6.7 (Hybritech, Tandem-E, Krefeld, Germany)) were still available from the "Assay Comparison Study for PSA" (11). The study was performed in accordance with practices and ethical standards of the Committee on Ethical Issues of the University of Münster and the Declaration of Helsinki including informed consent of the participants. Clinical and histological validation of the sera and sample processing were performed as previously described (6).

The 305 aliquots were used to determine PSA concentrations using the new modification of the ACS assay (ACS PSA, Bayer). Results were compared to the concentrations previously determined in identical samples (6) using the previous modification of the ACS assay (ACS PSA2, Chiron). Determinations of free and total PSA from an equimolar-response reference assay combination (Tandem- $R$ free and Tandem- $E$, Hybritech) were taken from the study mentioned above and used to study molar response characteristics as described previously (6). Clinical performance was evaluated by comparison of areas under ROC curves. (12). The ROC curves were obtained by plotting sensitivities (Y-axis) vs. 1-sensitivities (X-axis) using all measured PSA concentrations as cut-offs. In these ROC curves, an ideal diagnostic test yields an AUC of 1

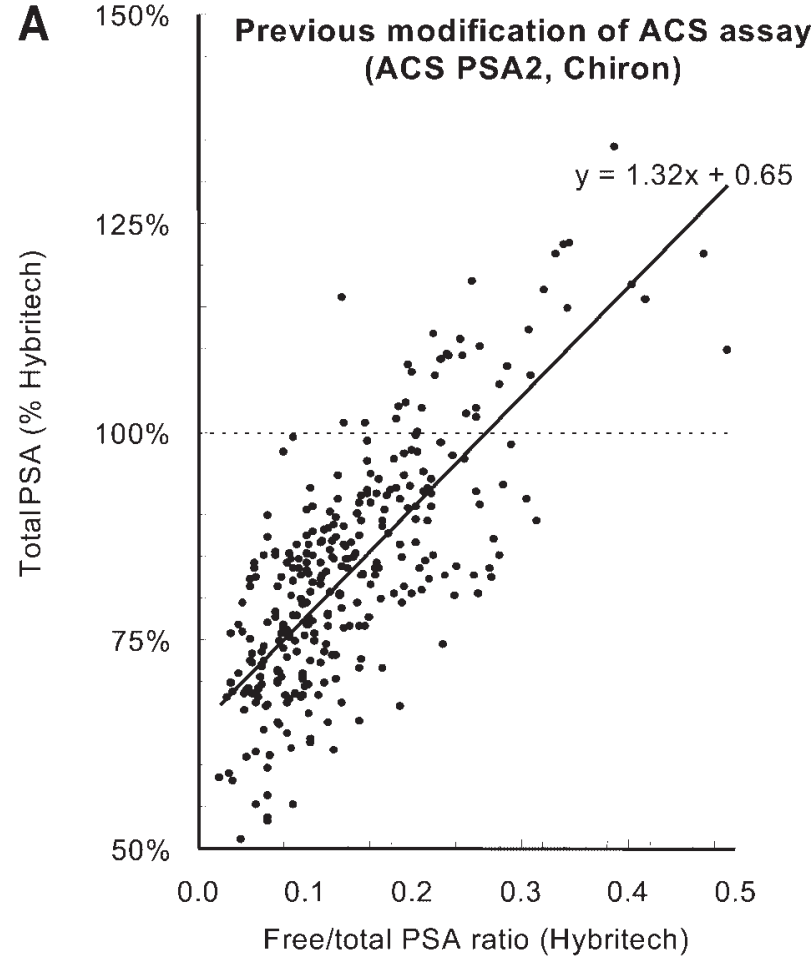

Figure 1 Equimolarity analysis for the previous (ACS PSA2, Chiron) (A) and new (ACS PSA, Bayer) (B) modification of the ACS assay for total PSA. Results are obtained from aliquots of identical serum samples of 150 patients with untreated PCA and 155 men with benign prostate hypertrophy. Total PSA concentrations ( $y$-axis) expressed as percent of the equimolar-response reference method (Hybritech, Tandem-E) are plotted against free/total PSA ratios (Hybritech Tandem- $R$

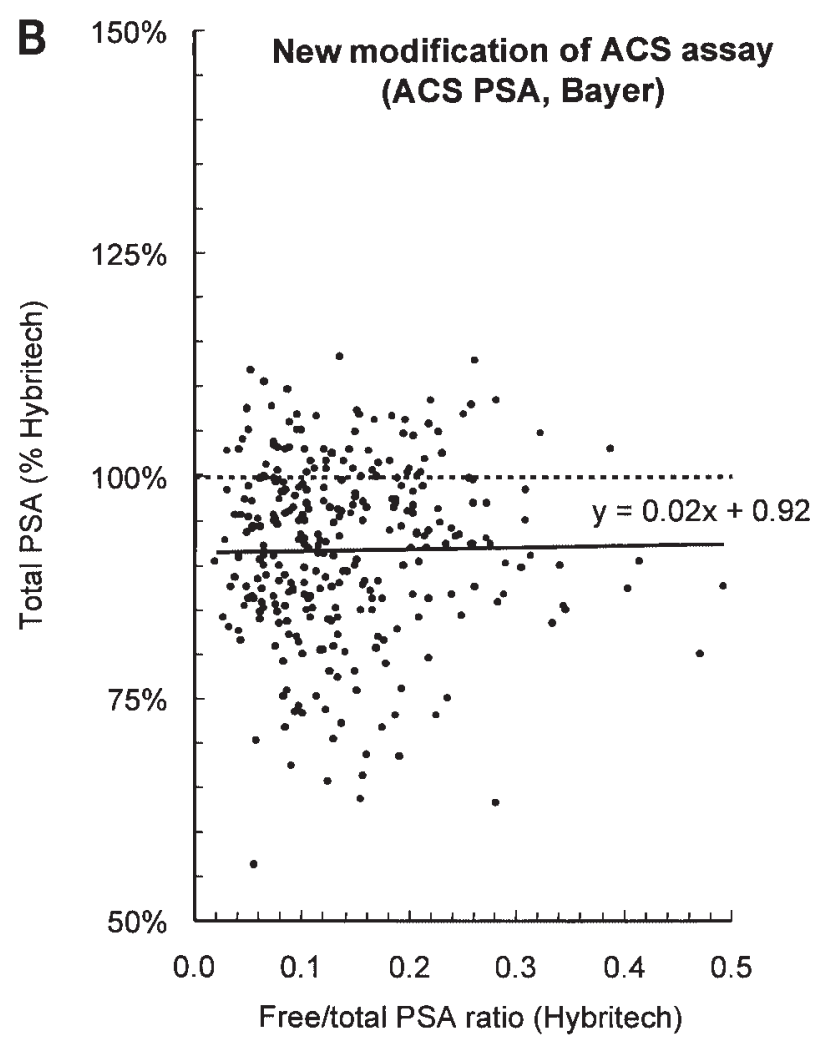

free/Tandem-E). The previous modification (ACS PSA2, Chiron) (A) yields a positive slope, indicating that free PSA is overreported (linear regression). The new modification (ACS PSA, Bayer) (B) results in a horizontal regression line running parallel to the dotted $100 \%$ reference line which indicates equimolar-response characteristics, on average reporting approximately $10 \%$ lower total PSA concentrations than the Tandem-E assay. 
Table 1 Comparison of total PSA (t-PSA) results from the nonequimolar (ACS PSA2, Chiron) and equimolar (ACS PSA,
Bayer) modifications of the ACS assay in different ranges of the free/total PSA ratio (f/t-PSA).

\begin{tabular}{|c|c|c|c|c|}
\hline f/t-PSA Hybritech & $\mathrm{n}$ & $\begin{array}{l}\text { Average t-PSA } \\
\text { Previous ACS modification } \\
\text { (ACS PSA2, Chiron) } \\
\text { [\% Hybritech] }\end{array}$ & $\begin{array}{l}\text { Average t-PSA } \\
\text { New ACS modification } \\
\text { (ACS PSA, Bayer) } \\
\text { [\% Hybritech] }\end{array}$ & $\begin{array}{l}\mathrm{p} \\
\text { (Student t-test) }\end{array}$ \\
\hline $0.000-0.099$ & 98 & $73 \%$ & $92 \%$ & $<0.001$ \\
\hline $0.100-0.199$ & 137 & $83 \%$ & $91 \%$ & $<0.001$ \\
\hline $0.200-0.299$ & 56 & $95 \%$ & $95 \%$ & 0.848 \\
\hline$>0.300$ & 14 & $114 \%$ & $91 \%$ & $<0.001$ \\
\hline Total & 305 & $83 \%$ & $92 \%$ & $<0.001$ \\
\hline
\end{tabular}

and a useless test an AUC of 0.5. For comparison of two methods, the hypothesis that the difference between the two AUCs is zero was statistically tested (MedCalc Version 6.0 software, www.medcalc.be). A p-value $<0.05$ was considered to indicate a significant difference in diagnostic performance.

\section{Results}

Total PSA concentrations ranged from $\geq 2$ to $\leq 30 \mu \mathrm{g} / \mathrm{l}$ by the Tandem-E assay. The free/total PSA ratio ranged from 0.02 to 0.49 (Tandem-R free/Tandem-E). The PSA concentrations ( $\mu \mathrm{g} / \mathrm{l}$, Hybritech, Tandem-E) in the 150 patients with PCA (mean 12.1; median 11.1; SD 6.7) and the 155 men without PCA (mean 6.1; median 3.7; SD 5.3) differed as expected.

As previously reported (6), the previous modification of the ACS assay (ACS PSA2, Chiron) showed a marked nonequimolar response as indicated by the skewed regression line in Figure 1A. The new modification of the ACS assay (ACS PSA, Bayer) was found to have an equimolar response represented by the horizontal regression line in Figure 1B. On average, the new ACS modification (ACS PSA, Bayer) yielded approximately $10 \%$ lower total PSA concentrations than the Tandem-E assay.

The new ACS modification (ACS PSA, Bayer) on average yielded slightly higher total PSA $(\mu \mathrm{g} / \mathrm{l})$ concentrations (mean 8.1, range 1.7-26.3, SD 5.7, median 6.3 (95\% $\mathrm{Cl} 5.7$ to 7.6$)$ ) than the previous modification (ACS PSA2, Chiron) (mean 7.6, range 1.3-35.8, SD 5.9, median 6.0 (95\% Cl 5.1 to 7.0$)$ ). The mean difference by paired Student t-test was significant ( $p<0.0001$ ). However, when the data comparing the ACS assays from Figures $1 \mathrm{~A}$ and $1 \mathrm{~B}$ are divided into four segments (depending on the free/total PSA ratio determined by the Hybritech reference assays), a striking pattern emerges (see Table 1). In the first segment (free/total PSA ratio between 0 and 0.099), the nonequimolar-response ACS assay (ACS PSA2, Chiron) underestimates total PSA values by an average of $-27 \%$, whereas the equimolarresponse ACS assay (ACS PSA, Bayer) underestimates total PSA values by an average of only $-8 \%(n=98$, $p<0.001$ ). Conversely, in the fourth segment (free/total PSA ratio $>0.3$ ), the nonequimolar-response ACS assay (ACS PSA2, Chiron) overestimates total PSA values by

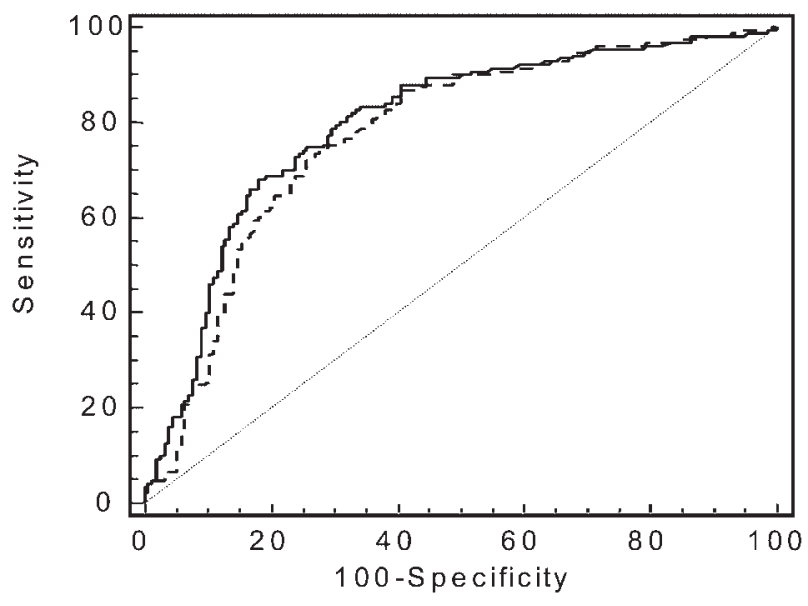

Figure 2 ROC curves of the previous (ACS PSA2, Chiron; dotted line) and new (ACS PSA, Bayer; solid line) modification of the ACS assay. New modification (ACS PSA, Bayer): AUC = 0.80 (95\% Cl: $0.75-0.84$ ) Previous modification (ACS PSA2, Chiron): $\mathrm{AUC}=0.77(95 \% \mathrm{Cl}: 0.72-0.82)$ Difference in AUC = 0.03 (95\% Cl: $0.01-0.04), p=0.01$ ( $p$-value is given for the tested hypothesis that the difference between the two AUCs is zero). The new ACS assay modification (ACS PSA, Bayer) corresponding to Figure $1 \mathrm{~B}$ resulted in equimolar assay characteristics with improved diagnostic performance $(p=0.01)$.

an average of $14 \%$, whereas the equimolar-response ACS assay (ACS PSA, Bayer) still underestimates total PSA values by an average of $-9 \%(n=14, p<0.001)$. These data support the concern that nonequimolar-response assays tend to underestimate total PSA in some men with PCA (especially those patients with low free/total PSA ratios in the first segment). These data also demonstrate how nonequimolar-response assays tend to overestimate total PSA in some men with benign prostates (i.e., patients with high free/total PSA ratios in the fourth segment).

Upon ROC analysis, the new modification (ACS PSA, Bayer) produced a small but statistically significant improvement of the AUC (Figure 2) in comparison to the previous modification (ACS PSA2, Chiron). The observed $3 \%$ gain in AUC is comparable to the AUC increases that Abbott ( $2 \%$ ) and bioMérieux (3\%) achieved when replacing their initially nonequimolar-response assays by an equimolar-response assay format as investigated in a slightly larger population (6). At $90 \%$ 
sensitivity, the specificity of the new modification (ACS PSA, Bayer) increased from $45 \%(37-53,95 \% \mathrm{Cl})$ to $48 \%(40-57)$, thus excluding six of the 155 men without PCA from biopsy without missing additional PCA. The cut-offs to achieve $90 \%$ sensitivity were 2.9 and $3.5 \mu \mathrm{g} / \mathrm{l}$ for the previous (ACS PSA2, Chiron) and new modification (ACS PSA, Bayer), respectively.

\section{Discussion}

Although, theoretically equimolar-response assays should allow a better discrimination between benign prostate hyperplasia and PCA, this was only recently demonstrated in a clinical setting (6). Responding to Graves' (1) and subsequently Sokoloff's (2) early recommendations, assay producers have withdrawn PSA assays from the market that did not exhibit equimolarresponse assay characteristics. As was expected (3, 13), assay suppliers who developed assays for free PSA, but whose total PSA assays were not equimolarresponse assays, made efforts to modify their total PSA assays. Consequently, nonequimolar-response assays have already been superseded or withdrawn (14) and it can be anticipated that more assay modifications will appear on the market (10).

A platform for the standardization of PSA assays (1, $2,15)$ may be achieved by the production of equimolarresponse PSA assays. However, recently Blijenberg et al. (16) investigated three equimolar-response assays before and after recalibration with two calibration standards and concluded that, even after recalibration, they were still not completely interchangeable. Despite the remaining problems in immunoassay standardization comprehensively reviewed by Stenman (17), it should be easier to standardize equimolar-response PSA assays or, as Stenman proposes, at least achieve a "harmonization" as an interim solution. A reduction in interassay variability may be accomplished (18) when calibrators made available recently (19) are applied to equimolar-response assays. Until PSA assay standardization is achieved, establishment of assay-specific reference ranges is strongly recommended to avoid misinterpretation of PSA concentrations $(10,13,20,21)$.

As the present study has shown, the new modification of the ACS assay (ACS PSA, Bayer) exhibits equimolar-response assay characteristics (Figure 1B) in concert with an improvement of diagnostic performance (Figure 2). Although the difference in AUC between the previous (ACS PSA2, Chiron) and new (ACS PSA, Bayer) ACS assay modifications is relatively small, it is statistically significant. These data provide additional evidence for clinical advantages of equimolar-response over nonequimolar-response PSA assay formats.

\section{Acknowledgements}

The PSA comparison study on which this evaluation is based was financially supported in equal parts by the producers of all the participating assays. The technical assistance of Francoise
Flammang, Beate Pepping-Schefers, Sabine Plonka, Karin Hofmann and Ashley Steele is gratefully acknowledged.

\section{References}

1. Graves HC. Issues on standardization of immunoassays for prostate-specific antigen: a review. Clin Invest Med 1993; 16:415-24.

2. Sokoloff RL, Wolfert RL, Rittenhouse HG. Standardization of PSA immunoasaays: proposals and practical limitations. J Clin Ligand Assay 1995; 18:86-92.

3. Semjonow A, Oberpenning F, Brandt B, Zechel C, Brandau $W$, Hertle L. Impact of free prostate-specific antigen on discordant measurement results of assays for total prostatespecific antigen. Urology 1996; 48:10-5.

4. Lilja H, Christensson A, Dahlen U, Matikainen MT, Nilsson $\mathrm{O}$, Pettersson $\mathrm{K}$, et al. Prostate-specific antigen in serum occurs predominantly in complex with alpha-1-antichymotrypsin. Clin Chem 1991; 37:1618-25.

5. Stenman UH, Leinonen J, Alfthan H, Rannikko S, Tuhkanen $\mathrm{K}$, Alfthan $\mathrm{O}$. A complex between prostate-specific antigen and alpha 1-antichymotrypsin is the major form of prostate-specific antigen in serum of patients with prostatic cancer: assay of the complex improves clinical sensitivity for cancer. Cancer Res 1991; 51:222-6.

6. Semjonow A, Oberpenning F, Weining C, Schön M, Brandt $B$, De Angelis G, et al. Do modifications of nonequimolar assays for total prostate-specific antigen improve detection of prostate cancer? Clin Chem 2001; 47:1472-5.

7. Strobel SA, Sokoloff RL, Wolfert RL, Rittenhouse HG. Multiple forms of prostate-specific antigen in serum measured differently in equimolar- and skewed-response assays. Clin Chem 1995; 41:125-6.

8. Zhou AM, Tewari PC, Bluestein BI, Caldwell GW, Larsen FL. Multiple forms of prostate-specific antigen in serum: differences in immunorecognition by monoclonal and polyclonal assays. Clin Chem 1993; 39:2483-91.

9. Bluestein BI, Tewari PC, Weitz S, Zhou A. Multiple forms of prostate-specific antigen in serum measured differently in equimolar- and skewed-response assays. Clin Chem 1995; 41:126-7.

10. Semjonow A, De Angelis G, Oberpenning F, Schmid HP, Brandt $B$, Hertle L. The clinical impact of different assays for prostate specific antigen. BJU Int 2000; 86:590-7.

11. Semjonow A, Weining C, Oberpenning F, Heinecke A, Terpe A, Schmid HP, et al. Application of assay-specific cutoff values: results of the assay comparison study for PSA. Eur Urol 1999; 35 (Suppl 2):18.

12. Hanley JA, McNeil BJ. A method of comparing the areas under receiver operating characteristic curves derived from the same cases. Radiology 1983; 148:839-43.

13. Semjonow A, Brandt B, Oberpenning F, Roth S, Hertle L. Discordance of assay methods creates pitfalls for the interpretation of prostate-specific antigen values. Prostate 1996; 7:3-16.

14. Ward AM, Catto JW, Hamdy FC. Prostate specific antigen: biology, biochemistry and available commercial assays. Ann Clin Biochem 2001; 38:633-51.

15. Graves HC. Standardization of immunoassays for prostate-specific antigen. A problem of prostate-specific antigen complexation or a problem of assay design? Cancer 1993; 72:3141-4.

16. Blijenberg BG, Storm BN, Kruger AE, Schröder FH. On the standardization of total prostate-specific antigen: an exercise with two reference preparations. Clin Chem Lab Med 1999; 37:545-52. 
17. Stenman UH. Immunoassay standardization: is it possible, who is responsible, who is capable? Clin Chem 2001; 47:815-20.

18. Chan DW, Sokoll LJ. WHO first international standards for prostate-specific antigen: the beginning of the end for assay discrepancies? Clin Chem 2000; 46:1291-2.

19. Rafferty B, Rigsby P, Rose M, Stamey T, Gaines Das R. Reference reagents for prostate-specific antigen (PSA): establishment of the first international standards for free PSA and PSA (90:10). Clin Chem 2000; 46:1310-7.

20. Blijenberg BG, Yurdakul G, Van Zelst BD, Bangma $\mathrm{CH}$, Wildhagen MF, Schröder FH. Discordant performance of assays for free and total prostate-specific antigen in relation to the early detection of prostate cancer. BJU Int 2001; $88: 545-50$.
21. Jung K, Lein M, Schnorr D, Brux B, Henke W, Loening S. Comparison between equimolar- and skewed-response assays of prostate specific antigen: is there an influence on the clinical significance when measuring total serum prostate specific antigen? Ann Clin Biochem 1996; 33:209-14.

Received 11 June 2002, revised 12 September 2002, accepted 4 October 2003

Corresponding author: Frank Oberpenning, M.D., University of Münster, Department of Urology, Albert-Schweitzer-Str. 33, 48129 Münster, Germany Phone: + 49 251-834 7443, Fax: + 49 251-834 8492

E-mail: oberpen@uni-muenster.de 